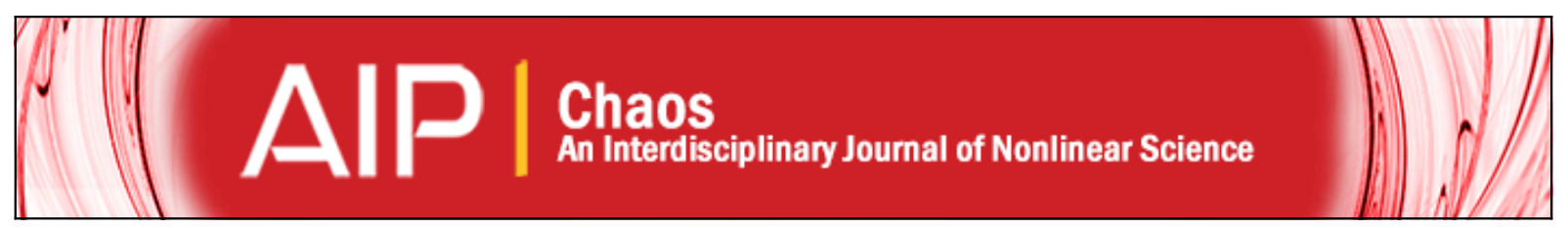

On the absence of analytic integrability of the Bianchi Class B cosmological models Antoni Ferragut, Jaume Llibre, and Chara Pantazi

Citation: Chaos: An Interdisciplinary Journal of Nonlinear Science 23, 013119 (2013); doi: 10.1063/1.4790828 View online: http://dx.doi.org/10.1063/1.4790828

View Table of Contents: http://scitation.aip.org/content/aip/journal/chaos/23/1?ver=pdfcov

Published by the AIP Publishing

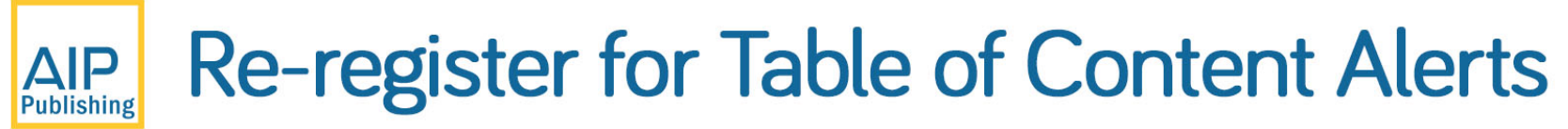




\title{
On the absence of analytic integrability of the Bianchi Class B cosmological models
}

\author{
Antoni Ferragut, ${ }^{1, a)}$ Jaume Llibre, $^{2, \mathrm{~b})}$ and Chara Pantazi ${ }^{3, \mathrm{c})}$ \\ ${ }^{1}$ Departament de Matemàtica Aplicada I, Universitat Politècnica de Catalunya, ETSEIB, Av. Diagonal, 647, \\ 08028 Barcelona, Catalonia, Spain \\ ${ }^{2}$ Departament de Matemàtiques, Universitat Autònoma de Barcelona, Edifici C, 08193 Bellaterra, Barcelona, \\ Catalonia, Spain \\ ${ }^{3}$ Departament de Matemàtica Aplicada I, Universitat Politècnica de Catalunya, (EPSEB), Av. Doctor \\ Marañón, 44-50, 08028 Barcelona, Spain
}

(Received 24 July 2012; accepted 4 January 2013; published online 15 February 2013)

We follow Bogoyavlensky's approach to deal with Bianchi class B cosmological models. We characterize the analytic integrability of such systems. (C) 2013 American Institute of Physics. [http://dx.doi.org/10.1063/1.4790828]

Bianchi models are cosmological models that describe space-times which are foliated by homogeneous hypersurfaces of constant time and are divided into two classes, Class A and Class B. There are many studies about the integrability of Class A. Here, we study the integrability of Class B. For the homogeneous cosmological models of Class B, Einstein's system of differential equations reduces to a dynamical system of dimension seven according to Bogoyavlensky's approach. We show that in order to study the integrability of such systems, it is sufficient to deal with homogeneous polynomial differential systems of dimension six. Concretely, Bianchi $V$ is the simplest model and can be written as a homogeneous polynomial differential system of degree 2 . Bianchi $I V$ is dealt as a homogeneous polynomial differential system of degree 3 and the rest of the models, Bianchis III, VI, and VII are of degree 5. Due to the fact that all Bianchi class $B$ models have been reduced to homogeneous polynomial differential systems, the study of their analytic integrability reduces to analyze their homogeneous polynomial first integrals. We show that Bianchi model $V$ admits polynomial first integral, and we prove that the corresponding homogeneous polynomial differential systems that represent models Bianchi IV, III, VI, and VII do not admit polynomial first integrals. The fact that these Bianchi models are not completely integrable with analytic first integrals facilitates that they can have chaotic behavior.

\section{INTRODUCTION AND STATEMENT OF THE RESULTS}

Einstein's equations relate the geometry of the spacetime with the properties of the matter which occupied it. The matter occupying the space-time is determined by the stress energy tensor of the matter. In our study, we follow ${ }^{3}$ and we consider the hydrodynamical tensor of the matter. We will work with an equation of state of matter of the form $p=k \varepsilon$,

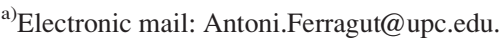

${ }^{b)}$ Electronic mail: jllibre@mat.uab.cat.

${ }^{\mathrm{c})}$ Electronic mail: chara.pantazi@upc.edu.
}

where $\varepsilon$ is the energy density of the matter, $p$ is the pressure and $0 \leq k<1$.

We can found in the literature some methods in order to construct some Hamiltonians for the Bianchi class B models, see, for example, Ref. 4 and references therein. Here, we follow Bogoyavlensky's approach, see Ref. 3. So according to Bogoyavlensky, for the homogeneous cosmological models of Class B Einstein's system of equations reduces to the following dynamical system in the phase space $p_{i}, q_{i}, p_{\varphi}, \varphi$, $i=1,2,3$,

$$
\begin{array}{ll}
\frac{d q_{i}}{d \tau}=\frac{\partial H}{\partial p_{i}}, & \frac{d p_{i}}{d \tau}=-\frac{\partial H}{\partial q_{i}}-h_{i}, \\
\frac{d \varphi}{d \tau}=\frac{\partial H}{\partial p_{\varphi}}, & \frac{d p_{\varphi}}{d \tau}=-\frac{\partial H}{\partial \varphi}-h_{\varphi}
\end{array}
$$

where the function $H$ is

$$
H=\frac{1}{\left(q_{1} q_{2} q_{3}\right)^{\frac{1-k}{2}}}\left(T\left(p_{i} q_{i}\right)+V_{G}\left(q_{i}\right)\right)
$$

with

$$
\begin{aligned}
T\left(p_{i}, q_{i}, p_{\varphi}\right) & =2 \sum_{1 \leq i<j \leq 3} p_{i} p_{j} q_{i} q_{j}-\sum_{i=1}^{3} p_{i}^{2} q_{i}^{2}-\frac{p_{\varphi}^{2} q_{1} q_{2}}{\left(n_{1} q_{1}-n_{2} q_{2}\right)^{2}}, \\
V_{G}\left(q_{i}\right) & =-\frac{1}{4}\left(12 a^{2} q_{1} q_{2}+\left(n_{1} q_{1}-n_{2} q_{2}\right)^{2}\right),
\end{aligned}
$$

and

$$
\begin{aligned}
& h_{1}=\frac{a^{2} q_{2}}{\left(q_{1} q_{2} q_{3}\right)^{\frac{1-k}{2}}}, \quad h_{2}=\frac{a^{2} q_{1}}{\left(q_{1} q_{2} q_{3}\right)^{\frac{1-k}{2}}}, \\
& h_{3}=\frac{-2 a^{2} q_{1} q_{2}}{q_{3}\left(q_{1} q_{2} q_{3}\right)^{\frac{1-k}{2}}}, \quad h_{\varphi}=\frac{a\left(n_{1} q_{1}-n_{2} q_{2}\right)^{2}}{\left(q_{1} q_{2} q_{3}\right)^{\frac{1-k}{2}}} .
\end{aligned}
$$

The constants $a \neq 0, n_{1}, n_{2}$ determine the type of model according to Table I, see also Refs. 1-3. System (1) in an explicit form writes as 
TABLE I. The classification of Bianchi class B cosmologies.

\begin{tabular}{lccccc}
\hline \hline Type & III & IV & V & VI & VII \\
\hline$a$ & 1 & 1 & 1 & $a \neq 1$ & $a$ \\
$n_{1}$ & 1 & 1 & 0 & 1 & 1 \\
$n_{2}$ & -1 & 0 & 0 & -1 & 1 \\
\hline \hline
\end{tabular}

$$
\begin{aligned}
\frac{d q_{1}}{d \tau}= & -2 q_{1}\left(q_{1} q_{2} q_{3}\right)^{(k-1) / 2}\left(p_{1} q_{1}+p_{2} q_{2}-p_{3} q_{3}\right), \\
\frac{d q_{2}}{d \tau}= & -2 q_{2}\left(q_{1} q_{2} q_{3}\right)^{(k-1) / 2}\left(p_{1} q_{1}+p_{3} q_{3}-p_{2} q_{2}\right), \\
\frac{d q_{3}}{d \tau}= & -2 q_{3}\left(q_{1} q_{2} q_{3}\right)^{(k-1) / 2}\left(p_{1} q_{1}+p_{2} q_{2}-p_{3} q_{3}\right), \\
\frac{d p_{1}}{d \tau}= & -\left(q_{1} q_{2} q_{3}\right)^{(k-1) / 2}\left(2 p_{1}\left(p_{2} q_{2}+p_{3} q_{3}-p_{1} q_{1}\right)\right. \\
& \left.+\frac{p_{\varphi}^{2}\left(n_{1} q_{1}+n_{2} q_{2}\right)}{\left(n_{1} q_{1}-n_{2} q_{2}\right)^{3}} q_{2}-\frac{1}{2} n_{1}\left(n_{1} q_{1}-n_{2} q_{2}\right)-2 a^{2} q_{2}\right) \\
& +\frac{1-k}{2 q_{1}} H \\
\frac{d p_{2}}{d \tau}= & -\left(q_{1} q_{2} q_{3}\right)^{(k-1) / 2}\left(2 p_{2}\left(p_{1} q_{1}+p_{3} q_{3}-p_{2} q_{2}\right)\right. \\
& \left.-\frac{p_{\varphi}^{2}\left(n_{1} q_{1}+n_{2} q_{2}\right)}{\left(n_{1} q_{1}-n_{2} q_{2}\right)^{3}} q_{1}-\frac{1}{2} n_{2}\left(n_{1} q_{1}-n_{2} q_{2}\right)-2 a^{2} q_{1}\right) \\
& +\frac{1-k}{2 q_{2}} H, \\
\frac{d p_{3}}{d \tau}= & -\left(q_{1} q_{2} q_{3}\right)^{(k-1) / 2} \\
& \times\left(2 p_{3}\left(p_{1} q_{1}+p_{2} q_{2}-p_{3} q_{3}\right)-2 a^{2} \frac{q_{1} q_{2}}{q_{3}}\right)+\frac{1-k}{2 q_{3}} H, \\
\frac{d \varphi}{d \tau}= & -\left(q_{1} q_{2} q_{3}\right)^{(k-1) / 2} \frac{2 q_{1} q_{2} p_{\varphi}}{\left(n_{1} q_{1}-n_{2} q_{2}\right)^{2}}, \\
\frac{d p_{\varphi}}{d \tau}= & -\left(q_{1} q_{2} q_{3}\right)^{(k-1) / 2} a\left(n_{1} q_{1}-n_{2} q_{2}\right)^{2}, \\
&
\end{aligned}
$$

with $\bar{H}=T+V_{G}$.

System (1) applies in the subspace defined by Einstein's equation

$$
R=p_{1} q_{1}+p_{2} q_{2}-2 p_{3} q_{3}+\frac{p_{\varphi}}{2 a}=0,
$$

for more details see Ref. 3. Additionally, if $\mathcal{X}$ is the associated vector field to system (3) we have that $\mathcal{X}(R)=0$ and so $R$ is a first integral of system (3). On the other hand, easy computations shows that

$$
\mathcal{X}(H)=-4 a^{2} \frac{\left(q_{1} q_{2} q_{3}\right)^{k}}{q_{3}} R
$$

and so the function $H$ given by Eq. (2) is a first integral of system (3) when this system is restricted to the hypersurface $R=0$.
We notice that for Bianchi $\mathrm{V}$ we have $p_{\varphi}=0$ and $\dot{\varphi}=0$, so for this model we obtain a simplest model of six equations. After the change of coordinates and time

$$
x_{i}=q_{i}, \quad x_{i+3}=p_{i} q_{i}, \quad i=1,2,3, \quad d \tau_{0}=\frac{\left(q_{1} q_{2} q_{3}\right)^{\frac{k-1}{2}}}{2} d \tau,
$$

the Bianchi V model writes as a homogeneous polynomial differential system of degree 2 :

$$
\begin{aligned}
& \dot{x}_{1}=x_{1}\left(-x_{4}+x_{5}+x_{6}\right), \\
& \dot{x}_{2}=x_{2}\left(x_{4}-x_{5}+x_{6}\right), \\
& \dot{x}_{3}=x_{3}\left(x_{4}+x_{5}-x_{6}\right), \\
& \dot{x}_{4}=x_{1} x_{2}+\frac{k-1}{4}\left(3 x_{1} x_{2}+\Lambda\right), \\
& \dot{x}_{5}=x_{1} x_{2}+\frac{k-1}{4}\left(3 x_{1} x_{2}+\Lambda\right), \\
& \dot{x}_{6}=x_{1} x_{2}+\frac{k-1}{4}\left(3 x_{1} x_{2}+\Lambda\right),
\end{aligned}
$$

where

$$
\Lambda=x_{4}^{2}+x_{5}^{2}+x_{6}^{2}-2\left(x_{4} x_{5}+x_{4} x_{6}+x_{5} x_{6}\right) .
$$

The condition stated in Eq. (4) reduces to $x_{4}+x_{5}-2 x_{6}=0$ for Bianchi V model. The function (2) becomes the first integral

$$
\left(x_{1} x_{2} x_{3}\right)^{\frac{k-1}{2}}\left(3 x_{1} x_{2}+\Lambda\right) \text {. }
$$

For Bianchi models III, IV, VI, and VII we notice that $n_{1}=1$. Moreover, system (3) is defined on the subspace (4), hence we can eliminate the momentum $p_{\varphi}$. So we can reduce the dimension of system (3) to seven. Note also that in order to study the integrability of system (3) we do not need to consider Eq. (7). In short, for studying the integrability of system (3), it is sufficient to deal with a differential system of dimension six.

For Bianchi IV after the change of coordinates and time

$$
\begin{gathered}
x_{i}=q_{i}, \quad x_{i+3}=p_{i} q_{i}, \quad i=1,2,3, \\
d \tau_{0}=\frac{q_{1}\left(q_{1} q_{2} q_{3}\right)^{\frac{k-1}{2}}}{2} d \tau,
\end{gathered}
$$

and the above considerations we get the six-dimensional homogeneous polynomial differential system of degree 3

$$
\begin{aligned}
& \dot{x}_{1}=x_{1}^{2}\left(-x_{4}+x_{5}+x_{6}\right), \\
& \dot{x}_{2}=x_{1} x_{2}\left(x_{4}-x_{5}+x_{6}\right), \\
& \dot{x}_{3}=x_{1} x_{3}\left(x_{4}+x_{5}-x_{6}\right), \\
& \dot{x}_{4}=\frac{x_{1}^{3}}{4}+x_{1}^{2} x_{2}-2 x_{2}\left(x_{4}+x_{5}-2 x_{6}\right)^{2}+\frac{k-1}{4} R, \\
& \dot{x}_{5}=x_{1}^{2} x_{2}+2 x_{2}\left(x_{4}+x_{5}-2 x_{6}\right)^{2}+\frac{k-1}{4} R, \\
& \dot{x}_{6}=x_{1}^{2} x_{2}+\frac{k-1}{4} R,
\end{aligned}
$$

where 


$$
R=x_{1}^{3} / 4+3 x_{1}^{2} x_{2}+4 x_{2}\left(x_{4}+x_{5}-2 x_{6}\right)^{2}+x_{1} \Lambda .
$$

The function (2) becomes the first integral

$$
\left(x_{1} x_{2} x_{3}\right)^{\frac{k-1}{2}}\left(\frac{x_{1}^{2}}{4}+3 x_{1} x_{2}+\frac{4 x_{2}\left(x_{4}+x_{5}-2 x_{6}\right)^{2}}{x_{1}}+\Lambda\right) \text {. }
$$

In the rest of the cases, i.e., for the Bianchi models III, VI, and VII after the change of coordinates and time

$$
\begin{gathered}
x_{i}=q_{i}, \quad x_{i+3}=p_{i} q_{i}, \quad i=1,2,3, \\
\frac{d \tau_{0}}{d \tau}=\frac{N^{3}\left(q_{1} q_{2} q_{3}\right)^{\frac{k-1}{2}}}{2},
\end{gathered}
$$

where $N=x_{1}-n_{2} x_{2}$, and the above considerations we obtain the six-dimensional homogeneous polynomial differential system of degree 5

$$
\begin{aligned}
\dot{x}_{1}= & x_{1} N^{3}\left(-x_{4}+x_{5}+x_{6}\right), \\
\dot{x}_{2}= & x_{2} N^{3}\left(x_{4}-x_{5}+x_{6}\right), \\
\dot{x}_{3}= & x_{3} N^{3}\left(x_{4}+x_{5}-x_{6}\right), \\
\dot{x}_{4}= & \frac{1}{4} x_{1} N^{4}+a^{2} x_{1} x_{2} N^{3}+\frac{k-1}{4} N S \\
& -2 a^{2} x_{1} x_{2}\left(x_{1}+n_{2} x_{2}\right)\left(x_{4}+x_{5}-2 x_{6}\right)^{2}, \\
\dot{x}_{5}= & \frac{1}{4} N^{5}-\frac{1}{4} x_{1} N^{4}+a^{2} x_{1} x_{2} N^{3}+\frac{k-1}{4} N S \\
& +2 a^{2} x_{1} x_{2}\left(x_{1}+n_{2} x_{2}\right)\left(x_{4}+x_{5}-2 x_{6}\right)^{2}, \\
\dot{x}_{6}= & a^{2} x_{1} x_{2} N^{3}+\frac{k-1}{4} N S,
\end{aligned}
$$

where

$$
S=N^{4} / 4+4 a^{2} x_{1} x_{2}\left(x_{4}+x_{5}-2 x_{6}\right)^{2}+N^{2}\left(3 a^{2} x_{1} x_{2}+\Lambda\right) .
$$

The function (2) becomes the first integral

$$
\left(x_{1} x_{2} x_{3}\right)^{\frac{k-1}{2}}\left(\frac{N^{2}}{4}+3 a^{2} x_{1} x_{2}+\Lambda+\frac{4 a^{2} x_{1} x_{2}\left(x_{4}+x_{5}-2 x_{6}\right)^{2}}{N^{2}}\right) .
$$

A study of the integrability of Bianchi models using its symmetries has been done in Ref. 5, but no information is given there on the analytic integrability of these systems. The analytic integrability of Class A has been studied in previous works by several authors..$^{5-10,12-17}$ Here, our aim is to study the analytic integrability of all Bianchi models of class $\mathrm{B}$ in the variables $\left(x_{1}, x_{2}, x_{3}, x_{4}, x_{5}, x_{6}\right)$. In our study, we will use the following result, see Ref. 12 .

Proposition 1. Let $F$ be an analytic function and let $F=\sum_{i} F_{i}$ be its decomposition into homogeneous polynomials $F_{i}$ of degree $i$. Then $F$ is an analytic first integral of a homogeneous differential system if and only if for all $i F_{i}$ is a homogeneous polynomial first integral of the homogeneous system.
Due to Proposition 1 and the fact that all Bianchi class B models have been reduced to homogeneous polynomial differential systems, see the systems (5), (7), and (8), the study of their analytic integrability reduces to analyze their homogeneous polynomial first integrals. Our main result is the following.

\section{Theorem 2. The following statements hold}

(a) System (5) has the two independent first integrals $x_{4}-$ $x_{5}$ and $x_{4}-x_{6}$. These two first integrals become the same when we restrict system (5) to (4), i.e. $x_{4}+x_{5}-2 x_{6}=0$, and any other polynomial first integral is a polynomial in the variable $x_{4}-x_{5}$.

(b) System (7) has no polynomial first integrals.

(c) Systems (8) have no polynomial first integrals.

Section II provides some technical lemmas that we will use for the proof of Theorem 2. In Sec. III, we prove the first statement of Theorem 2, namely, we study Bianchi V. In Sec. IV, we deal with Bianchi IV. The integrability of Bianchi III, VI, and VII is studied in Sec. V.

\section{SOME AUXILIARY RESULTS}

Lemma 3. Let $x_{k}$ be a one-dimensional variable, $k \in\{1, \ldots, n\}, n>1$ and let $f=f\left(x_{1}, \ldots, x_{n}\right)$ be a polynomial. For $l \in\{1, \ldots, n\}$ and $c_{0}$ a constant let $f_{l}=\left.f\left(x_{1}, \ldots, x_{n}\right)\right|_{x_{l}=c_{0}}$. Then there exists a polynomial $g=g\left(x_{1}, \ldots, x_{n}\right)$ such that $f=f_{l}+\left(x_{l}-c_{0}\right) g$ (see Ref. 11$)$.

The next two lemmas are proved in Ref. 8.

Lemma 4. Let $g=g\left(x_{4}, x_{5}, x_{6}\right)$ be a homogeneous polynomial solution of the homogeneous partial differential equation

$$
\left(a_{1} x_{4}+a_{2} x_{5}+a_{3} x_{6}\right) g+\frac{k-1}{4} \Lambda\left(\frac{\partial g}{\partial x_{4}}+\frac{\partial g}{\partial x_{5}}+\frac{\partial g}{\partial x_{6}}\right)=0,
$$

where $a_{1}, a_{2}, a_{3} \in \mathbb{R}$ are such that $\left(a_{1}-a_{2}\right)^{2}+\left(a_{1}-a_{3}\right)^{2}$ $\neq 0$. Then $g \equiv 0$.

Lemma 5. Let $g=g\left(x_{4}, x_{5}, x_{6}\right)$ and $h_{2}=h_{2}\left(x_{4}-x_{5}\right.$, $\left.x_{4}-x_{6}\right)$ be homogeneous polynomials of respective degrees $n-2$ and $n$ such that

$$
2\left(x_{4}-x_{5}+x_{6}\right) g+\frac{k-1}{4} \Lambda\left(\frac{\partial g}{\partial x_{4}}+\frac{\partial g}{\partial x_{5}}+\frac{\partial g}{\partial x_{6}}\right)+\frac{\partial h_{2}}{\partial x_{5}}=0 .
$$

Then $h_{2}=h_{2}\left(x_{4}-x_{6}\right)$ and $g \equiv 0$.

\section{PROOF OF STATEMENT (A) OF THEOREM 2}

It is clear that system (5) has the first integrals $x_{4}-x_{5}$ and $x_{4}-x_{6}$. We shall prove in this section that under the restriction $x_{4}+x_{5}-2 x_{6}=0$ system (5) has only one independent first integral and it is $x_{4}-x_{5}$.

Suppose that system (5) has a homogeneous polynomial first integral $h$ of degree $n$. According to Lemma 3 we can write $h=h_{1}\left(x_{2}, x_{3}, x_{4}, x_{5}, x_{6}\right)+x_{1}^{j} g_{1}\left(x_{1}, x_{2}, x_{3}, x_{4}, x_{5}, x_{6}\right)$, with $x_{1} \backslash g_{1}$ and $j \in \mathbb{N}$. Suppose that $g_{1} \not \equiv 0$. System (5) on $x_{1}=0$ writes 


$$
\begin{aligned}
& \dot{x}_{2}=x_{2}\left(x_{4}-x_{5}+x_{6}\right), \\
& \dot{x}_{3}=x_{3}\left(x_{4}+x_{5}-x_{6}\right) \\
& \dot{x}_{4}=\frac{k-1}{4} \Lambda, \\
& \dot{x}_{5}=\frac{k-1}{4} \Lambda, \\
& \dot{x}_{6}=\frac{k-1}{4} \Lambda .
\end{aligned}
$$

Since $h$ is a first integral of system (5), we have that $h_{1}$ is a first integral of system (11). System (11) admits the two polynomial first integrals $x_{4}-x_{5}$ and $x_{4}-x_{6}$ and the two non-polynomial first integrals

$$
x_{2}^{\frac{3}{2}(k-1)} \Lambda\left(\frac{x_{4}+x_{5}+x_{6}-2 \sqrt{\Delta}}{x_{4}+x_{5}+x_{6}+2 \sqrt{\Delta}}\right)^{\frac{x_{4}-2 x_{5}+x_{6}}{\sqrt{\Delta}}}
$$

and

$$
x_{3}^{\frac{3}{2}(k-1)} \Lambda\left(\frac{x_{4}+x_{5}+x_{6}-2 \sqrt{\Delta}}{x_{4}+x_{5}+x_{6}+2 \sqrt{\Delta}}\right)^{\frac{x_{4}+x_{5}-2 x_{6}}{\sqrt{\Delta}}},
$$

where

$$
\Delta=x_{4}^{2}+x_{5}^{2}+x_{6}^{2}-x_{4} x_{5}-x_{4} x_{6}-x_{5} x_{6} .
$$

As these four first integrals of system (11) are independent and $h_{1}$ is a polynomial first integral of Eq. (11), we get, taking into account the equality $x_{4}+x_{5}-2 x_{6}=0$, that $h_{1}=C_{1}\left(x_{4}-x_{5}\right)^{n}$, where $C_{1} \in \mathbb{R}$. Now since $h$ and $x_{4}-x_{5}$ are first integrals of system (5) we have that $x_{1}^{j} g_{1}$ is also a first integral of system (5). Therefore

$$
\begin{aligned}
& j\left(-x_{4}+x_{5}+x_{6}\right) g_{1}+x_{1}\left(-x_{4}+x_{5}+x_{6}\right) \frac{\partial g_{1}}{\partial x_{1}} \\
& +x_{2}\left(x_{4}-x_{5}+x_{6}\right) \frac{\partial g_{1}}{\partial x_{2}}+x_{3}\left(x_{4}+x_{5}-x_{6}\right) \frac{\partial g_{1}}{\partial x_{3}} \\
& +\left(x_{1} x_{2}+\frac{k-1}{4} \Lambda\right)\left(\frac{\partial g_{1}}{\partial x_{4}}+\frac{\partial g_{1}}{\partial x_{5}}+\frac{\partial g_{1}}{\partial x_{6}}\right)=0
\end{aligned}
$$

Let $\bar{g}_{1}=\left.g_{1}\right|_{x_{1}=0} \not \equiv 0$. On $x_{1}=0$ we have

$$
\begin{aligned}
& j\left(-x_{4}+x_{5}+x_{6}\right) \bar{g}_{1}+x_{2}\left(x_{4}-x_{5}+x_{6}\right) \frac{\partial \bar{g}_{1}}{\partial x_{2}} \\
& \quad+x_{3}\left(x_{4}+x_{5}-x_{6}\right) \frac{\partial \bar{g}_{1}}{\partial x_{3}}+\frac{k-1}{4} \Lambda\left(\frac{\partial \bar{g}_{1}}{\partial x_{4}}+\frac{\partial \bar{g}_{1}}{\partial x_{5}}+\frac{\partial \bar{g}_{1}}{\partial x_{6}}\right)=0 .
\end{aligned}
$$

Write $\bar{g}_{1}=x_{2}^{l} g_{2}$, with $l \in \mathbb{N} \cup\{0\}, x_{2} \nmid g_{2}$ and $g_{2} \not \equiv 0$. Then

$$
\begin{aligned}
& {\left[j\left(-x_{4}+x_{5}+x_{6}\right)+l\left(x_{4}-x_{5}+x_{6}\right)\right] g_{2}} \\
& \quad+x_{2}\left(x_{4}-x_{5}+x_{6}\right) \frac{\partial g_{2}}{\partial x_{2}}+x_{3}\left(x_{4}+x_{5}-x_{6}\right) \frac{\partial g_{2}}{\partial x_{3}} \\
& \quad+\frac{k-1}{4} \Lambda\left(\frac{\partial g_{2}}{\partial x_{4}}+\frac{\partial g_{2}}{\partial x_{5}}+\frac{\partial g_{2}}{\partial x_{6}}\right)=0 .
\end{aligned}
$$

Set $\bar{g}_{2}=\left.g_{2}\right|_{x_{2}=0} \not \equiv 0$. Then on $x_{2}=0$ we have

$$
\begin{aligned}
& {\left[j\left(-x_{4}+x_{5}+x_{6}\right)+l\left(x_{4}-x_{5}+x_{6}\right)\right] \bar{g}_{2}} \\
& \quad+x_{3}\left(x_{4}+x_{5}-x_{6}\right) \frac{\partial \bar{g}_{2}}{\partial x_{3}}+\frac{k-1}{4} \Lambda\left(\frac{\partial \bar{g}_{2}}{\partial x_{4}}+\frac{\partial \bar{g}_{2}}{\partial x_{5}}+\frac{\partial \bar{g}_{2}}{\partial x_{6}}\right)=0 .
\end{aligned}
$$

Now let $\bar{g}_{2}=x_{3}^{m} g_{3}$, with $m \in \mathbb{N} \cup\{0\}, x_{3} \nmid g_{3}$ and $g_{3} \not \equiv 0$. We have

$$
\begin{aligned}
& {\left[j\left(-x_{4}+x_{5}+x_{6}\right)+l\left(x_{4}-x_{5}+x_{6}\right)\right.} \\
& \left.\quad+m\left(x_{4}+x_{5}-x_{6}\right)\right] g_{3}+x_{3}\left(x_{4}+x_{5}-x_{6}\right) \frac{\partial g_{3}}{\partial x_{3}} \\
& \quad+\frac{k-1}{4} \Lambda\left(\frac{\partial g_{3}}{\partial x_{4}}+\frac{\partial g_{3}}{\partial x_{5}}+\frac{\partial g_{3}}{\partial x_{6}}\right)=0 .
\end{aligned}
$$

Let $\bar{g}_{3}=\left.g_{3}\right|_{x_{3}=0} \not \equiv 0$. On $x_{3}=0$ we obtain

$$
\begin{aligned}
& {\left[j\left(-x_{4}+x_{5}+x_{6}\right)+l\left(x_{4}-x_{5}+x_{6}\right)\right.} \\
& \left.\quad+m\left(x_{4}+x_{5}-x_{6}\right)\right] \bar{g}_{3}+\frac{k-1}{4} \Lambda\left(\frac{\partial \bar{g}_{3}}{\partial x_{4}}+\frac{\partial \bar{g}_{3}}{\partial x_{5}}+\frac{\partial \bar{g}_{3}}{\partial x_{6}}\right)=0 .
\end{aligned}
$$

We are under the hypotheses of Lemma 4, hence, we have $\bar{g}_{3} \equiv 0$, which is in contradiction with the assumptions. Therefore, $h=C_{1}\left(x_{4}-x_{5}\right)^{n}$ and the proof follows.

\section{PROOF OF STATEMENT (B) OF THEOREM 2}

We study in this section the analytic integrability of Bianchi model IV. We consider system (7). Let $h=h\left(x_{1}, \ldots, x_{6}\right)$ be a homogeneous polynomial first integral of degree $n$ of system (7). Using Lemma 3 we can write $h=h_{2}\left(x_{1}, x_{3}, \ldots, x_{6}\right)+x_{2}^{l} g_{2}\left(x_{1}, \ldots, x_{6}\right)$, with $l \in \mathbb{N}$ and $h_{2}$ and $g_{2}$ homogeneous polynomials such that $x_{2} \nmid g_{2}$. Assume that $g_{2} \not \equiv 0$. On $x_{2}=0$ system (7) becomes, after canceling a common factor $x_{1}$, doing a change in the independent variable,

$$
\begin{aligned}
& \dot{x}_{1}=x_{1}\left(-x_{4}+x_{5}+x_{6}\right), \\
& \dot{x}_{3}=x_{3}\left(x_{4}+x_{5}-x_{6}\right), \\
& \dot{x}_{4}=\frac{x_{1}^{2}}{4}+\frac{k-1}{4}\left(\frac{x_{1}^{2}}{4}+\Lambda\right), \\
& \dot{x}_{5}=\frac{k-1}{4}\left(\frac{x_{1}^{2}}{4}+\Lambda\right), \\
& \dot{x}_{6}=\frac{k-1}{4}\left(\frac{x_{1}^{2}}{4}+\Lambda\right) .
\end{aligned}
$$

We notice that $h_{2}$ is a first integral of system (13). We write $h_{2}=h_{3}\left(x_{3}, \ldots, x_{6}\right)+x_{1}^{j} g_{3}\left(x_{1}, x_{3}, \ldots, x_{6}\right)$, with $j \in \mathbb{N}$ and $h_{3}$ and $g_{3}$ homogeneous polynomials such that $x_{1} \Varangle g_{3}$. Assume that $g_{3} \not \equiv 0$. System (13) on $x_{1}=0$ writes

$$
\begin{aligned}
& \dot{x}_{3}=x_{3}\left(x_{4}+x_{5}-x_{6}\right), \\
& \dot{x}_{4}=\frac{k-1}{4} \Lambda, \\
& \dot{x}_{5}=\frac{k-1}{4} \Lambda, \\
& \dot{x}_{6}=\frac{k-1}{4} \Lambda .
\end{aligned}
$$


Straightforward computations show that system (14) has the three independent first integrals $x_{4}-x_{5}, x_{4}-x_{6}$ and

$$
x_{3}^{\frac{3}{2}(k-1)} \Lambda\left(\frac{x_{4}+x_{5}+x_{6}-2 \sqrt{\Delta}}{x_{4}+x_{5}+x_{6}+2 \sqrt{\Delta}}\right)^{\frac{x_{4}+x_{5}-2 x_{6}}{\sqrt{\Delta}}},
$$

where $\Delta$ is given by relation (12). As $h_{3}$ is a polynomial first integral of system (14), we have $h_{3}=h_{3}\left(x_{4}-x_{5}, x_{5}-x_{6}\right)$. The following lemma shows that indeed $h=h_{3}\left(x_{5}-x_{6}\right)$ $+x_{2}^{l} g_{2}\left(x_{1}, \ldots, x_{6}\right)$.

Lemma 6. We have that $h_{3}=h_{3}\left(x_{5}-x_{6}\right)$ and $g_{3} \equiv 0$.

Proof. As $h_{2}=h_{3}+x_{1}^{j} g_{3}$ is a first integral of system (13), we have

$$
\begin{aligned}
& x_{1}^{j}\left[j\left(-x_{4}+x_{5}+x_{6}\right) g_{3}+x_{1}\left(-x_{4}+x_{5}+x_{6}\right) \frac{\partial g_{3}}{\partial x_{1}}\right. \\
& \quad+x_{3}\left(x_{4}+x_{5}-x_{6}\right) \frac{\partial g_{3}}{\partial x_{3}}+\frac{x_{1}^{2}}{4} \frac{\partial g_{3}}{\partial x_{4}} \\
& \left.\quad+\frac{k-1}{4}\left(\frac{x_{1}^{2}}{4}+\Lambda\right)\left(\frac{\partial g_{3}}{\partial x_{4}}+\frac{\partial g_{3}}{\partial x_{5}}+\frac{\partial g_{3}}{\partial x_{6}}\right)\right]+\frac{x_{1}^{2}}{4} \frac{\partial h_{3}}{\partial x_{4}}=0 .
\end{aligned}
$$

We distinguish some cases depending on the value of $j$.

If $j=1$ then Eq. (15) becomes

$$
\begin{aligned}
& \left(-x_{4}+x_{5}+x_{6}\right) g_{3}+x_{1}\left(-x_{4}+x_{5}+x_{6}\right) \frac{\partial g_{3}}{\partial x_{1}} \\
& +x_{3}\left(x_{4}+x_{5}-x_{6}\right) \frac{\partial g_{3}}{\partial x_{3}}+\frac{x_{1}^{2}}{4} \frac{\partial g_{3}}{\partial x_{4}} \\
& \quad+\frac{k-1}{4}\left(\frac{x_{1}^{2}}{4}+\Lambda\right)\left(\frac{\partial g_{3}}{\partial x_{4}}+\frac{\partial g_{3}}{\partial x_{5}}+\frac{\partial g_{3}}{\partial x_{6}}\right)+\frac{x_{1}}{4} \frac{\partial h_{3}}{\partial x_{4}}=0 .
\end{aligned}
$$

Let $\bar{g}_{3}=\left.g_{3}\right|_{x_{1}=0} \not \equiv 0$. On $x_{1}=0$ we have

$$
\begin{gathered}
\left(-x_{4}+x_{5}+x_{6}\right) \bar{g}_{3}+x_{3}\left(x_{4}+x_{5}-x_{6}\right) \frac{\partial \bar{g}_{3}}{\partial x_{3}} \\
+\frac{k-1}{4} \Lambda\left(\frac{\partial \bar{g}_{3}}{\partial x_{4}}+\frac{\partial \bar{g}_{3}}{\partial x_{5}}+\frac{\partial \bar{g}_{3}}{\partial x_{6}}\right)=0 .
\end{gathered}
$$

Write $\bar{g}_{3}=x_{3}^{m} g_{4} \not \equiv 0$, with $m \in \mathbb{N} \cup\{0\}$ and $x_{3} \nmid g_{4}$. Then,

$$
\begin{aligned}
& {\left[\left(-x_{4}+x_{5}+x_{6}\right)+m\left(x_{4}+x_{5}-x_{6}\right)\right] g_{4}} \\
& \quad+x_{3}\left(x_{4}+x_{5}-x_{6}\right) \frac{\partial g_{4}}{\partial x_{3}}+\frac{k-1}{4} \Lambda\left(\frac{\partial g_{4}}{\partial x_{4}}+\frac{\partial g_{4}}{\partial x_{5}}+\frac{\partial g_{4}}{\partial x_{6}}\right)=0 .
\end{aligned}
$$

Let $\bar{g}_{4}=\left.g_{4}\right|_{x_{3}=0} \not \equiv 0$. On $x_{3}=0$ we have

$$
\begin{gathered}
{\left[\left(-x_{4}+x_{5}+x_{6}\right)+m\left(x_{4}+x_{5}-x_{6}\right)\right] \bar{g}_{4}} \\
+\frac{k-1}{4} \Lambda\left(\frac{\partial \bar{g}_{4}}{\partial x_{4}}+\frac{\partial \bar{g}_{4}}{\partial x_{5}}+\frac{\partial \bar{g}_{4}}{\partial x_{6}}\right)=0 .
\end{gathered}
$$

Applying Lemma 4 we obtain $\bar{g}_{4} \equiv 0$, which is a contradiction. Hence $g_{3} \equiv 0$. Back to Eq. (15) we have $\partial h_{3} / \partial x_{4} \equiv 0$, which means that $h_{3}=h_{3}\left(x_{5}-x_{6}\right)$. Then the lemma follows in the case $j=1$.
If $j>2$, then from Eq. (15) we have that $x_{1} \mid\left(\partial h_{3} / \partial x_{4}\right)$ and thus $\partial h_{3} / \partial x_{4} \equiv 0$. Now we can proceed as in the case $j=1$ to obtain the equation

$$
\begin{gathered}
{\left[j\left(-x_{4}+x_{5}+x_{6}\right)+m\left(x_{4}+x_{5}-x_{6}\right)\right] \bar{g}_{4}} \\
+\frac{k-1}{4} \Lambda\left(\frac{\partial \bar{g}_{4}}{\partial x_{4}}+\frac{\partial \bar{g}_{4}}{\partial x_{5}}+\frac{\partial \bar{g}_{4}}{\partial x_{6}}\right)=0 .
\end{gathered}
$$

Applying Lemma 4 we arrive to a contradiction and hence $g_{3} \equiv 0$. The lemma follows in the case $j>2$.

If $j=2$ then Eq. (15) writes

$$
\begin{aligned}
& 2\left(-x_{4}+x_{5}+x_{6}\right) g_{3}+x_{1}\left(-x_{4}+x_{5}+x_{6}\right) \frac{\partial g_{3}}{\partial x_{1}} \\
& \quad+x_{3}\left(x_{4}+x_{5}-x_{6}\right) \frac{\partial g_{3}}{\partial x_{3}}+\frac{x_{1}^{2}}{4} \frac{\partial g_{3}}{\partial x_{4}} \\
& \quad+\frac{k-1}{4}\left(\frac{x_{1}^{2}}{4}+\Lambda\right)\left(\frac{\partial g_{3}}{\partial x_{4}}+\frac{\partial g_{3}}{\partial x_{5}}+\frac{\partial g_{3}}{\partial x_{6}}\right)+\frac{1}{4} \frac{\partial h_{3}}{\partial x_{4}}=0 .
\end{aligned}
$$

Let $\bar{g}_{3}=\left.g_{3}\right|_{x_{1}=0} \not \equiv 0$. On $x_{1}=0$ we have

$$
\begin{aligned}
& 2\left(-x_{4}+x_{5}+x_{6}\right) \bar{g}_{3}+x_{3}\left(x_{4}+x_{5}-x_{6}\right) \frac{\partial \bar{g}_{3}}{\partial x_{3}} \\
& +\frac{k-1}{4} \Lambda\left(\frac{\partial \bar{g}_{3}}{\partial x_{4}}+\frac{\partial \bar{g}_{3}}{\partial x_{5}}+\frac{\partial \bar{g}_{3}}{\partial x_{6}}\right)+\frac{1}{4} \frac{\partial h_{3}}{\partial x_{4}}=0 .
\end{aligned}
$$

Write $\bar{g}_{3}=x_{3}^{m} g_{4} \not \equiv 0$, with $m \in \mathbb{N} \cup\{0\}$ and $x_{3} \backslash g_{4}$. Then we get

$$
\begin{aligned}
& x_{3}^{m}\left[\left[2\left(-x_{4}+x_{5}+x_{6}\right)+m\left(x_{4}+x_{5}-x_{6}\right)\right] g_{4}\right. \\
& \quad+x_{3}\left(x_{4}+x_{5}-x_{6}\right) \frac{\partial g_{4}}{\partial x_{3}} \\
& \left.\quad+\frac{k-1}{4} \Lambda\left(\frac{\partial g_{4}}{\partial x_{4}}+\frac{\partial g_{4}}{\partial x_{5}}+\frac{\partial g_{4}}{\partial x_{6}}\right)\right]+\frac{1}{4} \frac{\partial h_{3}}{\partial x_{4}}=0 .
\end{aligned}
$$

If $m>0$ then $x_{3} \mid\left(\partial h_{3} / \partial x_{4}\right)$. Hence $\partial h_{3} / \partial x_{4} \equiv 0$ and $h_{3}=h_{3}\left(x_{5}-x_{6}\right)$. Let $\bar{g}_{4}=\left.g_{4}\right|_{x_{3}=0} \not \equiv 0$. On $x_{3}=0$ we obtain

$$
\begin{gathered}
{\left[2\left(-x_{4}+x_{5}+x_{6}\right)+m\left(x_{4}+x_{5}-x_{6}\right)\right] \bar{g}_{4}} \\
+\frac{k-1}{4} \Lambda\left(\frac{\partial \bar{g}_{4}}{\partial x_{4}}+\frac{\partial \bar{g}_{4}}{\partial x_{5}}+\frac{\partial \bar{g}_{4}}{\partial x_{6}}\right)=0 .
\end{gathered}
$$

Applying Lemma 4 we get a contradiction, hence we have $g_{3} \equiv 0$.

In the case $m=0$, let $\bar{g}_{4}=\left.g_{4}\right|_{x_{3}=0} \not \equiv 0$. On $x_{3}=0$ we obtain

$$
\begin{aligned}
& 2\left(-x_{4}+x_{5}+x_{6}\right) \bar{g}_{4}+\frac{k-1}{4} \Lambda\left(\frac{\partial \bar{g}_{4}}{\partial x_{4}}+\frac{\partial \bar{g}_{4}}{\partial x_{5}}+\frac{\partial \bar{g}_{4}}{\partial x_{6}}\right) \\
& \quad+\frac{1}{4} \frac{\partial h_{3}}{\partial x_{4}}=0 .
\end{aligned}
$$

We can apply Lemma 5 , permuting the variables $x_{4}$ by $x_{5}$, and we get that $h_{3}=h_{3}\left(x_{5}-x_{6}\right)$ so $\partial h_{3} / \partial x_{4} \equiv 0$. Now applying Lemma 4 we obtain $\bar{g}_{4} \equiv 0$. Hence the lemma follows in the case $j=2$.

After Lemma 6 we have that $h=h_{3}\left(x_{5}-x_{6}\right)$ $+x_{2}^{l} g_{2}\left(x_{1}, \ldots, x_{6}\right)$, with $l \in \mathbb{N}$ and $x_{2} \nmid g_{2}$. We note that $h_{3}$ is 
a homogeneous polynomial, hence, we can write $h_{3}\left(x_{5}-x_{6}\right)$ $=C_{3}\left(x_{5}-x_{6}\right)^{n}$, with $C_{3} \in \mathbb{R}$.

We recall that $h$ is a first integral of system (7). Thus it satisfies the equation

$$
\begin{aligned}
& x_{2}^{l}\left[l x_{1}\left(x_{4}-x_{5}+x_{6}\right) g_{2}+x_{1}^{2}\left(-x_{4}+x_{5}+x_{6}\right) \frac{\partial g_{2}}{\partial x_{1}}\right. \\
& \quad+x_{1} x_{2}\left(x_{4}-x_{5}+x_{6}\right) \frac{\partial g_{2}}{\partial x_{2}}+x_{1} x_{3}\left(x_{4}+x_{5}-x_{6}\right) \frac{\partial g_{2}}{\partial x_{3}} \\
& \quad+\frac{k-1}{4} R\left(\frac{\partial g_{2}}{\partial x_{4}}+\frac{\partial g_{2}}{\partial x_{5}}+\frac{\partial g_{2}}{\partial x_{6}}\right) \\
& \quad+\left(\frac{x_{1}^{3}}{4}+x_{1}^{2} x_{2}-2 x_{2}\left(x_{4}+x_{5}-2 x_{6}\right)^{2}\right) \frac{\partial g_{2}}{\partial x_{4}} \\
& \left.\quad+\left(x_{1}^{2} x_{2}+2 x_{2}\left(x_{4}+x_{5}-2 x_{6}\right)^{2}\right) \frac{\partial g_{2}}{\partial x_{5}}+x_{1}^{2} x_{2} \frac{\partial g_{2}}{\partial x_{6}}\right] \\
& \quad+2 C_{3} n x_{2}\left(x_{4}+x_{5}-2 x_{6}\right)^{2}\left(x_{5}-x_{6}\right)^{n-1}=0 .
\end{aligned}
$$

The following lemma ends the proof of statement (b) of Theorem 2, as it shows that $h \equiv 0$.

Lemma 7. We have that $h_{3} \equiv 0$ and $g_{2} \equiv 0$.

Proof. Suppose that $g_{2} \not \equiv 0$. We distinguish two cases depending on the value of $l$. If $l>1$ then from equation (16) we must take $C_{3}=0$ and therefore $h_{3} \equiv 0$. Let $\bar{g}_{2}=\left.g_{2}\right|_{x_{2}=0}$ $\not \equiv 0$. After simplifying $x_{2}^{l}$, equation (16) on $x_{2}=0$ becomes, after cancelling a common factor $x_{1}$,

$$
\begin{gathered}
l\left(x_{4}-x_{5}+x_{6}\right) \bar{g}_{2}+x_{1}\left(-x_{4}+x_{5}+x_{6}\right) \frac{\partial \bar{g}_{2}}{\partial x_{1}} \\
+x_{3}\left(x_{4}+x_{5}-x_{6}\right) \frac{\partial \bar{g}_{2}}{\partial x_{3}}+\frac{x_{1}^{2}}{4} \frac{\partial \bar{g}_{2}}{\partial x_{4}} \\
+\frac{k-1}{4}\left(\frac{x_{1}^{2}}{4}+\Lambda\right)\left(\frac{\partial \bar{g}_{2}}{\partial x_{4}}+\frac{\partial \bar{g}_{2}}{\partial x_{5}}+\frac{\partial \bar{g}_{2}}{\partial x_{6}}\right)=0 .
\end{gathered}
$$

Write $\bar{g}_{2}=x_{1}^{j} g_{3} \not \equiv 0$, with $j \in \mathbb{N} \cup\{0\}$ and $x_{1} \nmid g_{3}$. We get

$$
\begin{aligned}
& {\left[j\left(-x_{4}+x_{5}+x_{6}\right)+l\left(x_{4}-x_{5}+x_{6}\right)\right] g_{3}} \\
& \quad+x_{1}\left(-x_{4}+x_{5}+x_{6}\right) \frac{\partial g_{3}}{\partial x_{1}}+x_{3}\left(x_{4}+x_{5}-x_{6}\right) \frac{\partial g_{3}}{\partial x_{3}}+\frac{x_{1}^{2}}{4} \frac{\partial g_{3}}{\partial x_{4}} \\
& \quad+\frac{k-1}{4}\left(\frac{x_{1}^{2}}{4}+\Lambda\right)\left(\frac{\partial g_{3}}{\partial x_{4}}+\frac{\partial g_{3}}{\partial x_{5}}+\frac{\partial g_{3}}{\partial x_{6}}\right)=0 .
\end{aligned}
$$

Let $\bar{g}_{3}=\left.g_{3}\right|_{x_{1}=0} \not \equiv 0$. Then, on $x_{1}=0$ we have

$$
\begin{aligned}
& {\left[j\left(-x_{4}+x_{5}+x_{6}\right)+l\left(x_{4}-x_{5}+x_{6}\right)\right] \bar{g}_{3}} \\
& \quad+x_{3}\left(x_{4}+x_{5}-x_{6}\right) \frac{\partial \bar{g}_{3}}{\partial x_{3}}+\frac{k-1}{4} \Lambda\left(\frac{\partial \bar{g}_{3}}{\partial x_{4}}+\frac{\partial \bar{g}_{3}}{\partial x_{5}}+\frac{\partial \bar{g}_{3}}{\partial x_{6}}\right)=0 .
\end{aligned}
$$

Now write $\bar{g}_{3}=x_{3}^{m} g_{4} \not \equiv 0$, with $m \in \mathbb{N} \cup\{0\}$ and $x_{3} \nmid g_{4}$. We get

$$
\begin{aligned}
& {\left[j\left(-x_{4}+x_{5}+x_{6}\right)+l\left(x_{4}-x_{5}+x_{6}\right)\right.} \\
& \left.\quad+m\left(x_{4}+x_{5}-x_{6}\right)\right] g_{4}+x_{3}\left(x_{4}+x_{5}-x_{6}\right) \frac{\partial g_{4}}{\partial x_{3}} \\
& \quad+\frac{k-1}{4} \Lambda\left(\frac{\partial g_{4}}{\partial x_{4}}+\frac{\partial g_{4}}{\partial x_{5}}+\frac{\partial g_{4}}{\partial x_{6}}\right)=0 .
\end{aligned}
$$

Let $\bar{g}_{4}=\left.g_{4}\right|_{x_{3}=0} \not \equiv 0$. Then, on $x_{3}=0$ we have

$$
\begin{aligned}
& {\left[j\left(-x_{4}+x_{5}+x_{6}\right)+l\left(x_{4}-x_{5}+x_{6}\right)+m\left(x_{4}+x_{5}-x_{6}\right)\right] \bar{g}_{4}} \\
& \quad+\frac{k-1}{4} \Lambda\left(\frac{\partial \bar{g}_{4}}{\partial x_{4}}+\frac{\partial \bar{g}_{4}}{\partial x_{5}}+\frac{\partial \bar{g}_{4}}{\partial x_{6}}\right)=0 .
\end{aligned}
$$

Applying Lemma 4 we obtain $\bar{g}_{4} \equiv 0$, a contradiction. Hence $g_{2} \equiv 0$ and the lemma follows in the case $l>1$.

If $l=1$ then we can cancel a common factor $x_{2}$ in Eq. (16). Let $\bar{g}_{2}=\left.g_{2}\right|_{x_{2}=0} \not \equiv 0$. On $x_{2}=0$ Eq. (16) becomes

$$
\begin{aligned}
& x_{1}\left[\left(x_{4}-x_{5}+x_{6}\right) \bar{g}_{2}+x_{1}\left(-x_{4}+x_{5}+x_{6}\right) \frac{\partial \bar{g}_{2}}{\partial x_{1}}\right. \\
& +x_{3}\left(x_{4}+x_{5}-x_{6}\right) \frac{\partial \bar{g}_{2}}{\partial x_{3}}+\frac{x_{1}^{2}}{4} \frac{\partial \bar{g}_{2}}{\partial x_{4}} \\
& \left.\quad+\frac{k-1}{4}\left(\frac{x_{1}^{2}}{4}+\Lambda\right)\left(\frac{\partial \bar{g}_{2}}{\partial x_{4}}+\frac{\partial \bar{g}_{2}}{\partial x_{5}}+\frac{\partial \bar{g}_{2}}{\partial x_{6}}\right)\right] \\
& \quad+2 C_{3} n\left(x_{4}+x_{5}-2 x_{6}\right)^{2}\left(x_{5}-x_{6}\right)^{n-1}=0 .
\end{aligned}
$$

Clearly we must take $C_{3}=0$, and hence $h_{3} \equiv 0$. Then the equation writes

$$
\begin{aligned}
& \left(x_{4}-x_{5}+x_{6}\right) \bar{g}_{2}+x_{1}\left(-x_{4}+x_{5}+x_{6}\right) \frac{\partial \bar{g}_{2}}{\partial x_{1}} \\
& +x_{3}\left(x_{4}+x_{5}-x_{6}\right) \frac{\partial \bar{g}_{2}}{\partial x_{3}}+\frac{x_{1}^{2}}{4} \frac{\partial \bar{g}_{2}}{\partial x_{4}} \\
& +\frac{k-1}{4}\left(\frac{x_{1}^{2}}{4}+\Lambda\right)\left(\frac{\partial \bar{g}_{2}}{\partial x_{4}}+\frac{\partial \bar{g}_{2}}{\partial x_{5}}+\frac{\partial \bar{g}_{2}}{\partial x_{6}}\right)=0 .
\end{aligned}
$$

Write now $\bar{g}_{2}=x_{1}^{j} g_{3} \not \equiv 0$, with $j \in \mathbb{N} \cup\{0\}$ and $x_{1} \backslash g_{3}$. Then the above equation becomes

$$
\begin{aligned}
& {\left[j\left(-x_{4}+x_{5}+x_{6}\right)+\left(x_{4}-x_{5}+x_{6}\right)\right] g_{3}} \\
& \quad+x_{1}\left(-x_{4}+x_{5}+x_{6}\right) \frac{\partial g_{3}}{\partial x_{1}}+\frac{x_{1}^{2}}{4} \frac{\partial g_{3}}{\partial x_{4}} \\
& \quad+x_{3}\left(x_{4}+x_{5}-x_{6}\right) \frac{\partial g_{3}}{\partial x_{3}} \\
& \quad+\frac{k-1}{4}\left(\frac{x_{1}^{2}}{4}+\Lambda\right)\left(\frac{\partial g_{3}}{\partial x_{4}}+\frac{\partial g_{3}}{\partial x_{5}}+\frac{\partial g_{3}}{\partial x_{6}}\right)=0 .
\end{aligned}
$$

Let $\bar{g}_{3}=\left.g_{3}\right|_{x_{1}=0} \not \equiv 0$. Then, on $x_{1}=0$ we have

$$
\begin{aligned}
& {\left[j\left(-x_{4}+x_{5}+x_{6}\right)+\left(x_{4}-x_{5}+x_{6}\right)\right] \bar{g}_{3}} \\
& \quad+x_{3}\left(x_{4}+x_{5}-x_{6}\right) \frac{\partial \bar{g}_{3}}{\partial x_{3}}+\frac{k-1}{4} \Lambda\left(\frac{\partial \bar{g}_{3}}{\partial x_{4}}+\frac{\partial \bar{g}_{3}}{\partial x_{5}}+\frac{\partial \bar{g}_{3}}{\partial x_{6}}\right)=0 .
\end{aligned}
$$

Now write $\bar{g}_{3}=x_{3}^{m} g_{4} \not \equiv 0$, with $m \in \mathbb{N} \cup\{0\}$ and $x_{3} \nmid g_{4}$. We get

$$
\begin{aligned}
& {\left[j\left(-x_{4}+x_{5}+x_{6}\right)+\left(x_{4}-x_{5}+x_{6}\right)\right.} \\
& \left.\quad+m\left(x_{4}+x_{5}-x_{6}\right)\right] g_{4}+x_{3}\left(x_{4}+x_{5}-x_{6}\right) \frac{\partial g_{4}}{\partial x_{3}} \\
& \quad+\frac{k-1}{4} \Lambda\left(\frac{\partial g_{4}}{\partial x_{4}}+\frac{\partial g_{4}}{\partial x_{5}}+\frac{\partial g_{4}}{\partial x_{6}}\right)=0 .
\end{aligned}
$$

Let $\bar{g}_{4}=\left.g_{4}\right|_{x_{3}=0} \not \equiv 0$. Then, on $x_{3}=0$ we have 


$$
\begin{aligned}
& {\left[j\left(-x_{4}+x_{5}+x_{6}\right)+\left(x_{4}-x_{5}+x_{6}\right)\right.} \\
& \left.\quad+m\left(x_{4}+x_{5}-x_{6}\right)\right] \bar{g}_{4}+\frac{k-1}{4} \Lambda\left(\frac{\partial \bar{g}_{4}}{\partial x_{4}}+\frac{\partial \bar{g}_{4}}{\partial x_{5}}+\frac{\partial \bar{g}_{4}}{\partial x_{6}}\right)=0 .
\end{aligned}
$$

Applying Lemma 4 we obtain $\bar{g}_{4} \equiv 0$, a contradiction. Hence $g_{2} \equiv 0$ and the lemma follows also in the case $l=1$.

We have proved that the first integral $h$ is identically zero. Therefore, the system cannot have a polynomial first integral. Statement (b) of Theorem 2 follows.

\section{PROOF OF STATEMENT (C) OF THEOREM 2}

We study in this section the analytic integrability of Bianchi models III, VI, and VII.

Consider system (8) and let $h=h\left(x_{1}, \ldots, x_{6}\right)$ be a homogeneous polynomial first integral of degree $n$ of this system. Applying Lemma 3 we can write $h=h_{1}\left(x_{2}, \ldots, x_{6}\right)$ $+x_{1}^{j} g_{1}\left(x_{1}, \ldots, x_{6}\right)$, with $j \in \mathbb{N}$ and $h_{1}$ and $g_{1}$ homogeneous polynomials such that $x_{1} \nmid g_{1}$.

Assume that $g_{1} \not \equiv 0$. On $x_{1}=0$ system (8) becomes, after canceling a common factor $-n_{2} x_{2}^{3}$,

$$
\begin{aligned}
& \dot{x}_{2}=x_{2}\left(x_{4}-x_{5}+x_{6}\right), \\
& \dot{x}_{3}=x_{3}\left(x_{4}+x_{5}-x_{6}\right), \\
& \dot{x}_{4}=\frac{k-1}{4}\left(\frac{x_{2}^{2}}{4}+\Lambda\right), \\
& \dot{x}_{5}=\frac{x_{2}^{2}}{4}+\frac{k-1}{4}\left(\frac{x_{2}^{2}}{4}+\Lambda\right), \\
& \dot{x}_{6}=\frac{k-1}{4}\left(\frac{x_{2}^{2}}{4}+\Lambda\right) .
\end{aligned}
$$

We note that $h_{1}$ is a first integral of system (20). The following lemma provides more information about the expression of $h_{1}$.

Lemma 8. We have $h_{1}=h_{1}\left(x_{4}-x_{6}\right)$.

Proof. Swapping $x_{1}$ and $x_{2}$ and swapping $x_{4}$ and $x_{5}$ system (20) is system (13), for which we have proved that there is no other polynomial first integral than 0 , see Lemma 6. Then the lemma follows.

After Lemma 8 we have that $h=h_{1}\left(x_{4}-x_{6}\right)$ $+x_{1}^{j} g_{1}\left(x_{1}, \ldots, x_{6}\right)$, with $j \in \mathbb{N}$ and $x_{1} \Varangle g_{1}$. We recall that $h$ is a first integral of system (8). Thus it satisfies the equation

$$
\begin{aligned}
& x_{1}^{j}\left[j N^{3}\left(-x_{4}+x_{5}+x_{6}\right) g_{1}+x_{1} N^{3}\left(-x_{4}+x_{5}+x_{6}\right) \frac{\partial g_{1}}{\partial x_{1}}+x_{2} N^{3}\left(x_{4}-x_{5}+x_{6}\right) \frac{\partial g_{1}}{\partial x_{2}}+x_{3} N^{3}\left(x_{4}+x_{5}-x_{6}\right) \frac{\partial g_{1}}{\partial x_{3}}\right. \\
& \quad+\frac{k-1}{4} N S\left(\frac{\partial g_{1}}{\partial x_{4}}+\frac{\partial g_{1}}{\partial x_{5}}+\frac{\partial g_{1}}{\partial x_{6}}\right)+\left(\frac{1}{4} x_{1} N^{4}+a^{2} x_{1} x_{2} N^{3}-2 a^{2} x_{1} x_{2}\left(x_{1}+n_{2} x_{2}\right)\left(x_{4}+x_{5}-2 x_{6}\right)^{2}\right) \frac{\partial g_{1}}{\partial x_{4}} \\
& \left.\quad+\left(\frac{1}{4} N^{5}-\frac{1}{4} x_{1} N^{4}+a^{2} x_{1} x_{2} N^{3}+2 a^{2} x_{1} x_{2}\left(x_{1}+n_{2} x_{2}\right)\left(x_{4}+x_{5}-2 x_{6}\right)^{2}\right) \frac{\partial g_{1}}{\partial x_{5}}+a^{2} x_{1} x_{2} N^{3} \frac{\partial g_{1}}{\partial x_{6}}\right]+a^{2} x_{1} x_{2} N^{3} \frac{\partial h_{1}}{\partial x_{6}} \\
& \quad+\left(\frac{1}{4} x_{1} N^{4}+a^{2} x_{1} x_{2} N^{3}-2 a^{2} x_{1} x_{2}\left(x_{1}+n_{2} x_{2}\right)\left(x_{4}+x_{5}-2 x_{6}\right)^{2}\right) \frac{\partial h_{1}}{\partial x_{4}}=0 .
\end{aligned}
$$

The following lemma ends the proof of statement (c) of Theorem 2 , as it shows that $h \equiv 0$.

Lemma 9. We have that $h_{1} \equiv 0$ and $g_{1} \equiv 0$.

Proof. Write $h_{1}\left(x_{4}-x_{6}\right)=C_{1}\left(x_{4}-x_{6}\right)^{n}$, with $C_{1} \in \mathbb{R}$. Suppose that $g_{1} \not \equiv 0$. We distinguish two cases depending on the value of $j$. If $j>1$ then from Eq. (21) we have that $x_{1}$ divides

$$
C_{1} n\left(\frac{x_{2}^{4}}{4}-2 a^{2} n_{2} x_{2}^{2}\left(x_{4}+x_{5}-2 x_{6}\right)^{2}\right) .
$$

Hence, we must take $C_{2}=0$ and therefore $h_{2} \equiv 0$. Let $\bar{g}_{1}=\left.g_{1}\right|_{x_{1}=0} \not \equiv 0$. Equation (21) on $x_{1}=0$ becomes, after cancelling a common factor $-n_{2} x_{2}^{3}$,

$$
\begin{aligned}
& j\left(-x_{4}+x_{5}+x_{6}\right) \bar{g}_{1}+x_{2}\left(x_{4}-x_{5}+x_{6}\right) \frac{\partial \bar{g}_{1}}{\partial x_{2}} \\
& +x_{3}\left(x_{4}+x_{5}-x_{6}\right) \frac{\partial \bar{g}_{1}}{\partial x_{3}}+\frac{x_{2}^{2}}{4} \frac{\partial \bar{g}_{1}}{\partial x_{5}} \\
& \quad+\frac{k-1}{4}\left(\frac{x_{2}^{2}}{4}+\Lambda\right)\left(\frac{\partial \bar{g}_{1}}{\partial x_{4}}+\frac{\partial \bar{g}_{1}}{\partial x_{5}}+\frac{\partial \bar{g}_{1}}{\partial x_{6}}\right)=0 .
\end{aligned}
$$

Swapping $x_{1}$ and $x_{2}$ and swapping $x_{4}$ and $x_{5}$ we obtain Eq. (17). Hence we have $g_{1} \equiv 0$ and the lemma follows in the case $j>1$.

If $j=1$ then we can cancel a common factor $x_{1}$ in equation (21). Let $\bar{g}_{1}=\left.g_{1}\right|_{x_{1}=0} \not \equiv 0$. On $x_{1}=0$ Eq. (21) becomes, after cancelling a common factor $-n_{2} x_{2}^{2}$,

$$
\begin{aligned}
& x_{2}\left[\left(-x_{4}+x_{5}+x_{6}\right) \bar{g}_{1}+x_{2}\left(x_{4}-x_{5}+x_{6}\right) \frac{\partial \bar{g}_{1}}{\partial x_{2}}\right. \\
& \quad+x_{3}\left(x_{4}+x_{5}-x_{6}\right) \frac{\partial \bar{g}_{1}}{\partial x_{3}}+\frac{x_{2}^{2}}{4} \frac{\partial \bar{g}_{1}}{\partial x_{5}} \\
& \left.\quad+\frac{k-1}{4}\left(\frac{x_{2}^{2}}{4}+\Lambda\right)\left(\frac{\partial \bar{g}_{1}}{\partial x_{4}}+\frac{\partial \bar{g}_{1}}{\partial x_{5}}+\frac{\partial \bar{g}_{1}}{\partial x_{6}}\right)\right] \\
& \quad+C_{1} n\left(2 a^{2}\left(x_{4}+x_{5}-2 x_{6}\right)^{2}-\frac{n_{2}}{4} x_{2}^{2}\right)\left(x_{4}-x_{6}\right)^{n-1}=0 .
\end{aligned}
$$

Clearly we must take $C_{1}=0$, and hence $h_{1} \equiv 0$. Once again swapping $x_{1}$ and $x_{2}$ and swapping $x_{4}$ and $x_{5}$ this equation is Eq. (18). Hence, we have $g_{1} \equiv 0$ and the lemma follows in the case $j=1$. 


\section{DISCUSSION}

In this work we have proved that the Bianchi model $\mathrm{V}$ of Class B is partially integrable with analytic first integrals, but not completely integrable, and that the Bianchi models III, IV, VI, and VII of Class B have no analytic first integrals. Consequently, the partial integrability of the model $\mathrm{V}$ and the non-analytic integrability of the other Bianchi models of class B facilitates that they can have chaotic behavior.

\section{ACKNOWLEDGMENTS}

All the authors were partially supported by the MICINN/ FEDER grant MTM2008-03437. A.F. was additionally partially supported by grants Juan de la Cierva, 2009SGR410 and MTM2009-14163-C02-02. J.L. was additionally partially supported by an AGAUR Grant No. 2009SGR410, by ICREA Academia and by FP7-PEOPLE-2012-IRSES-316338. C.P. was additionally partially supported by the MICINN/FEDER Grant Nos. MTM2009-06973 and MTM2012-31714 and by an AGAUR Grant No. 2009SGR859.
${ }^{1}$ L. Bianchi, Mem. Math. Fis. Soc. Ital. Sci 11, 267-352 (1898).

${ }^{2} \mathrm{~L}$. Bianchi, Lezioni sulla teoria dei gruppi continui finiti di trasformazioni (Lectures on the theory of finite continuous transformation groups) (Spoerri, Pisa, 1918), pp. 550-557.

${ }^{3}$ O. I. Bogoyavlensky, Qualitative Theory of Dynamical Systems in Astrophysics and Gas Dynamics (Springer-Verlag, 1985).

${ }^{4}$ T. Christodoulakis, E. Korfiatis, and A. Paschos, Phys. Rev. D 54, 26912698 (1996); Erratum, Phys. Rev. D 56, 5279-5280 (1997).

${ }^{5}$ S. Cotsakis, P. G. L. Leach, and Ch. Pantazi, Gravitation Cosmol. 4, 314325 (1998).

${ }^{6}$ R. Cushman and J. Sniatycki, Rep. Math. Phys. 36, 75-89 (1995).

${ }^{7}$ A. Ferragut, J. Llibre, and Ch. Pantazi, J. Geom. Phys. 62, 381-386 (2012).

${ }^{8}$ A. Ferragut, J. Llibre, and Ch. Pantazi, Chaos, Solitons Fractals 48, 12-21 (2013).

${ }^{9}$ A. Latifi, M. Musette, and R. Conte, Phys. Lect. A 194, 83-92 (1994).

${ }^{10}$ J. Llibre and C. Valls, J.Math. Phys. 47, 022704-15 (2006).

${ }^{11}$ J. Llibre and C. Valls, J. Math. Phys. 51, 092702-13 (2010).

${ }^{12}$ J. Llibre and X. Zhang, Nonlinearity 15, 1269-1280 (2002).

${ }^{13}$ A. J. Maciejewski, J. Strelcyn, and M. Szydłowski, J. Math. Phys. 42, 1728-13 (2001)

${ }^{14}$ A.J. Maciejewski and M. Szydłowski, J. Phys. A 31, 2031-2043 (1998).

${ }^{15}$ A. J. Maciejewski and M. Szydłowski, Celestial Mech. Dyn. Astron. 73, 17-24 (1999).

${ }^{16}$ M. Szydłowski and M. Biesiada, J. Nonlinear Math. Phys. 9, 1-10 (2002).

${ }^{17}$ M. Szydłowski and J. Demaret, Gen. Rel. Grav. 31, 897-911 (1999). 